\title{
TRES NUEVOS REGISTROS PARA MÉXICO DE PLANTAS ACUÁTICAS VASCULARES
}

\author{
Alejandro Novelo R. \\ Instituto de Biología \\ Universidad Nacional Autónoma de México \\ Apartado postal 70-233 \\ 04510 México, D.F. \\ Y \\ JOHN H. WiERSEMA \\ Systematic Botany and Mycology Laboratory \\ United States Department of Agriculture \\ Beltsville Agricultural Research Center \\ Beltsville, Maryland, 20705-2350
}

\section{RESUMEN}

Se registran por primera vez para México, en particular del Istmo de Tehuantepec, Oaxaca, tres especies de plantas acuáticas vasculares, a mencionar: Bergia capensis L. (Elatinaceae), Eichhornia paniculata (Sprengel) Solms-Laub. (Pontederiaceae) y Nymphaea novogranatensis Wiersema (Nymphaeaceae).

\section{ABSTRACT}

Bergia capensis L. (Elatinaceae), Eichhornia paniculata (Sprengel) Solms-Laub. (Pontederiaceae), and Nymphaea novogranatensis Wiersema (Nymphaeaceae), three species of aquatic vascular plants, are reported for the first time from Mexico, all of them growing in the Isthmus of Tehuantepec area, state of Oaxaca.

Durante varios viajes de colecta al Istmo de Tehuantepec en las cercanías del puerto de Salina Cruz, en el estado de Oaxaca, tuvimos la oportunidad de colectar plantas acuáticas dulceacuícolas en sitios muy interesantes y de inundación efímera, entre las cuales, aparecieron tres nuevos registros para la flora acuática mexicana: Bergia capensis L., Eichhornia paniculata (Sprengel) Solms-Laub. y Nymphaea novogranatensis Wiersema.

De acuerdo con Zizumbo y Colunga (1982), quienes han trabajado en esta región ocupada por los indios Huaves, al este de Salina Cruz se ubica la Barra de San Mateo, en la cual se encuentran lagunas, esteros y depresiones topográficas llamadas localmente "bajiales" (wüeik en lengua huave). Estos ambientes se encuentran a lo largo de la barra 
formando un sistema de "vasos comunicantes", que corren de este a oeste dándole a la primera una ondulación característica. Así, los terrenos están catalogados localmente según su topografía en: bajiales (wüeik), que son superficies cóncavas que se inundan cada año y en donde el agua se acumula durante algún tiempo; tierras bajas (nagmiek iet), las que se anegan periódicamente pero en donde el agua no permanece ya que no forman una concavidad, así como las tierras colgadas (rondon iet) que presentan una suave pendiente y se inundan esporádicamente en sus partes altas y, con más frecuencia, en las partes bajas.

Estos vasos comunicantes, de acuerdo con Zizumbo y Colunga (1982), comienzan a llenarse entre septiembre y octubre durante el corto periodo de lluvias y también pueden llegar a ser alimentados por el río Tehuantepec. Además, temporalmente tienen una influencia de agua salina a través de la Laguna Quirio, la cual se llena de agua de mar a partir de la primera quincena de abril hasta fines de junio, época en que ya está casi seca.

El clima de la región, de acuerdo con la carta de climas (Anónimo, 1970), basada en la clasificación de Köppen modificada por García (1964) es del tipo Aw”, (w)ig, que corresponde al más seco de los cálido subhúmedos con lluvias en verano. La precipitación pluvial anual varía entre 900 y $1040 \mathrm{~mm}$. La evaporación total anual es 3.7 veces mayor que la precipitación total. La vegetación original fue modificada sustancialmente desde hace mucho tiempo y las especies arbóreas que hemos encontrado como dominantes en los bordes de las zonas que se inundan, derivan de los bosques tropicales caducifolios, entre las que destacan Parkinsonia aculeata y Prosopis juliflora.

Las visitas que se realizaron con la finalidad de colectar las plantas acuáticas de esta región, fueron hechas hacia el final de la época de lluvias (septiembre-octubre) durante varios años. El nivel de inundación varió considerablemente de un año a otro. El tiempo que permanecen anegados de agua dulce estos vasos comunicantes y zonas encharcadas aledañas, es de unas pocas semanas y, en algunos años secos, las plantas acuáticas no alcanzan a desarrollarse completamente, por lo que antes del comienzo de la floración el agua prácticamente ya desapareció. De ahí la importancia de colectar en estos ecosistemas acuáticos efímeros, que muchas veces sustentan una flora hidrófila muy característica, adaptada a condiciones ambientales severas.

A continuación se detallan en orden alfabético las tres especies que resultaron ser nuevos registros para la flora acuática mexicana.

Bergia capensis. Pertenece a la familia Elatinaceae, compuesta por dos géneros: Bergia y Elatine y por 36 a 40 especies, las cuales usualmente crecen en ambientes acuáticos dulceacuícolas (Molau, 1983; Cook, 1990). El género Bergia es cosmopolita y comprende aproximadamente 25 especies, que en su mayoría se encuentran en África. Hasta antes de este nuevo registro, de México solamente se conocía a Bergia texana (Hook.) Seub. de Baja California, Baja California Sur, Nuevo León (Lot et al., 1986; 1999) y Sinaloa.

Bergia capensis L., Mant. PI. Alt. 241. 1771.

Hierba acuática glabra, anual; tallos erectos, suculentos, de color rojizo, con raíces adventicias, comúnmente ramificándose en la base, de hasta $40 \mathrm{~cm}$ de alto y $1 \mathrm{~cm}$ de ancho; 
hojas opuestas, cortamente pecioladas, peciolos de 0.5 a $0.9 \mathrm{~mm}$ de largo; láminas elípticas, de 1 a $3 \mathrm{~cm}$ de largo y de 0.6 a $1.1 \mathrm{~cm}$ de ancho, margen serrado, base decurrente, ápice agudo; inflorescencia axilar, glomerulada, con 8-12 flores; flores hermafroditas, actinomorfas, cortamente pediceladas; pedicelos hasta de $1.5 \mathrm{~mm}$ de longitud; sépalos 5 , lanceolados, acrescentes, de color verde y con el borde rosado, hasta de $1.6 \mathrm{~mm}$ largo; pétalos 5 , lanceolados, de color blanco, de ca. $2 \mathrm{~mm}$ de largo; estambres 10, exsertos; filamentos lineal-lanceolados, membranáceos, aplanados, de ca. $1.8 \mathrm{~mm}$ de largo; anteras versátiles, circulares, de ca. $0.2 \mathrm{~mm}$ de largo y $0.2 \mathrm{~mm}$ de ancho; ovario súpero, globoso, 5-carpelar; estilos 5, lineares; estigma apical; fruto una cápsula 5-valvada, esférica, ligeramente comprimida, de $0.25 \mathrm{~mm}$ de alto y $0.3 \mathrm{~mm}$ de diámetro, de color rojizo; semillas numerosas, botuliformes, alveoladas, ligeramente recurvadas, de $0.5 \mathrm{~mm}$ de largo y $0.2 \mathrm{~mm}$ de ancho, de color moreno claro. Foto 1.

Ejemplares de respaldo: Oaxaca: municipio San Mateo del Mar, laguna Uderrop Piquil, on south side of road to San Mateo del Mar, $1.6 \mathrm{~km}$ east of junction with road to Colonia Cuauhtemoc, 17 September 1993, A. Novelo \& J. Wiersema 1193 (MEXU); municipio de San Mateo del Mar, $2 \mathrm{~km}$ al norte de la colonia Cuauhtémoc rumbo a Salina Cruz, aproximadamente $17 \mathrm{~km}$ al este de Salina Cruz, 17 de octubre de 1994, A. Novelo, J. Wiersema, B. Hellquist \& C. Horn 1274 (MEXU).

Planta propia de charcos y planos inundables efímeros que se forman entre los matorrales espinosos dominados por Parkinsonia aculeata. Alt. $5 \mathrm{~m}$. Se le encontró asociada a Echinodorus andrieuxii, Nymphaea ampla, N. pulchella y Heteranthera limosa. Especie originaria del Viejo Mundo, seguramente introducida en América y ahora probablemente diseminadas sus semillas por aves acuáticas a lo largo del trópico americano. Se le ha registrado de Costa Rica, Perú, Ecuador y de la isla de Cuba. Especie escasa y muy localizada en los sitios donde se desarrolla. Se puede considerar como amenazada por el peligro de que su hábitat desaparezca por las actividades humanas.

A continuación se anexa una clave para las especies de Bergia que se desarrollan en México y que puede resultar útil en su identificación.

1 Plantas glabras, tallos suculentos, rojizos

B. capensis

1 Plantas glandular-pubescentes, tallos no suculentos, verdosos

B. texana

Eichhornia paniculata. Pertenece a la familia Pontederiaceae, la cual está constituida por 5 a 9 géneros y aproximadamente 34 especies, propias de hábitats dulceacuícolas; se distribuyen preferentemente en zonas bajas y cálidas de ambos hemisferios (Novelo, 1996). El género Eichhornia tiene una distribución pantropical, pero en el continente americano se encuentra el mayor número de sus representantes. Hasta antes de este nuevo registro, de México solamente se conocían tres especies (Lot et al., 1986).

Eichhornia paniculata (Sprengel) Solms-Laub. in A. DC., Monogr. Phan. 4(2): 530. 1883.

Hierba acuática anual, de hasta $1.5 \mathrm{~m}$ de alto; tallos rizomatosos, muy reducidos; hojas emergentes, basales, peciolos hasta de $80 \mathrm{~cm}$ de largo, rectos; láminas ovadas a 
ampliamente ovadas, de 5 a $9 \mathrm{~cm}$ de largo y 2 a $5 \mathrm{~cm}$ de ancho, margen entero, base cordada, ápice agudo a acuminado; inflorescencia una espiga de espigas, con 10 a 35 flores, sésiles a subpediceladas, pedúnculo de 5 a $20 \mathrm{~cm}$ de largo, glabro a glandular-puberulento; espata inferior semejante en forma y tamaño a la lámina de las hojas no floríferas; espata superior de 1.5 a $3 \mathrm{~cm}$ de largo, abierta desde la base, ápice mucronulado; flores hermafroditas, zigomorfas, de ca. $1.8 \mathrm{~cm}$ de largo; perianto 6-lobulado, azulado, externamente glandular-puberulento, lóbulos con el margen entero; estambres 6, heterodínamos, insertos a distintas alturas, filamentos lineares, glandular-puberulentos, anteras versátiles, monomorfas, de 1.1 a $1.4 \mathrm{~mm}$ de largo; pistilo con nectarios septales; ovario súpero, 3-carpelar; estilo glandular-puberulento, tristílico; estigma capitado; fruto una cápsula 3-locular, dehiscente, madurando fuera del agua, de 0.7 a $1 \mathrm{~cm}$ de largo; semillas numerosas, elípticas, longitudinalmente acostilladas, de 0.7 a $1 \mathrm{~mm}$ de largo y 0.5 a $0.75 \mathrm{~mm}$ de ancho. Foto 2.

Ejemplares de respaldo: Oaxaca: municipio San Mateo del Mar, temporary fresh water pool west side of small mountain, east of road to Colonia Cuauhtemoc, $1.1 \mathrm{~km}$ South from road to San Mateo del Mar, 17 September 1993, A. Novelo \& J. Wiersema 1195 (MEXU); municipio San Mateo del Mar, $1 \mathrm{~km}$ al oeste de la colonia Cuauhtémoc, rumbo a Salina Cruz, aproximadamente $17 \mathrm{~km}$ al este de Salina Cruz, 12 de diciembre de 1994, A. Novelo, T. Philbrick, G. Crow y N. Oropeza 1404 (MEXU).

Planta propia de charcos efímeros que se forman en las partes bajas y abiertas de los matorrales espinosos dominados por Parkinsonia aculeata. Alt. $5 \mathrm{~m}$. Se le encontró asociada a Nymphaea novogranatensis y Marsilea deflexa. Especie americana que se conocía solamente de Nicaragua en Centroamérica, de Brasil hasta el norte de Argentina y en las Antillas Mayores. Introducida en Florida. Elemento escaso y muy localizado en los sitios donde se desarrolla. Se puede considerar como amenazada por el peligro de que su hábitat desaparezca por las actividades humanas.

A continuación se anexa una clave de las especies de Eichhornia que se desarrollan en México que puede resultar útil en su identificación.

1 Hojas arrosetadas, frecuentemente con los peciolos inflados; espata inferior con la lámina reducida y de menor tamaño que las hojas no floríferas E. crassipes 1 Hojas no arrosetadas, con los peciolos no inflados; espata inferior con la lámina similar a la de las hojas no floríferas.

2 Plantas anuales, con los tallos erectos; base de la lámina cordada

2 Plantas perennes, con los tallos rastreros, flotando sobre la superficie del agua; base de la lámina cuneada, atenuada u obtusa.

3 Pedúnculo glandular-puberulento; margen de los lóbulos internos del perianto fimbriado-ciliado E. azurea

3 Pedúnculo glabro; margen de los lóbulos del perianto entero 
Nymphaea novogranatensis. Pertenece a la familia Nymphaeaceae, la cual está compuesta por seis géneros y un poco más de 60 especies que crecen en ambientes acuáticos dulceacuícolas (Cook, 1990). El género Nymphaea es cosmopolita y comprende cerca de 40 especies con numerosos híbridos y variedades; muchos de ellos han sido utilizados como plantas ornamentales por la belleza de sus flores (Novelo y Bonilla, 1999). El género está dividido en cinco subgéneros de los cuales tres habitan en México. Nymphaea novogranatensis pertenece al subgénero Hydrocallis, el que se distingue entre otras cosas porque sus flores son nocturnas (Wiersema, 1987). El subgénero Lotos también tiene el mismo carácter, pero su área de distribución no llega al territorio de la República. En la última década se han dado a conocer para México dos nuevos registros de componentes de Hydrocallis: N. amazonum (Ramírez-García y Novelo, 1987) y N. prolifera (Olvera y Lot, 1991). Con la presente adición, ahora se sabe que existen en el país cinco especies pertenecientes a este subgénero.

Nymphaea novogranatensis Wiersema, Brittonia 36: 217. 1984.

Hierba acuática anual; rizoma tuberoso, tubérculo esférico a cilíndrico, negruzco; hojas dispuestas en espiral, largamente pecioladas, peciolo de hasta $35 \mathrm{~cm}$ de largo; láminas suborbiculares de hasta $14 \mathrm{~cm}$ de largo y $11 \mathrm{~cm}$ de ancho, margen entero, base cordada, ápice redondeado a ligeramente mucronulado; haz comúnmente de color verde o a veces ligeramente café-rojizo, con numerosas manchas purpúreas, en particular en la inserción con el peciolo; envés café-rojizo con múltiples manchas de color oscuro; nervios poco prominentes sin formar el patrón en forma de tela de araña; flores actinomorfas, perfectas, nocturnas, largamente pedunculadas; pedúnculos de hasta $30 \mathrm{~cm}$ de largo; sépalos externamente purpúreos, no variegados, ovado-lanceolados, de ca. $5 \mathrm{~cm}$ de largo y ca. 2 $\mathrm{cm}$ de ancho, con el ápice agudo a acuminado; pétalos 16(20), distribuidos en verticilos de 4, blanco-amarillentos, lanceolados, de ca. $4 \mathrm{~cm}$ de largo y $1.3 \mathrm{~cm}$ de ancho, con el ápice agudo a acuminado; estambres numerosos, distribuidos espiralmente en varias series, de 1 a $2.5 \mathrm{~cm}$ de largo, filamentos lineares, de hasta $1.3 \mathrm{~cm}$ de largo y $0.9 \mathrm{~mm}$ de ancho, anteras amarillas, de hasta $1.2 \mathrm{~cm}$ de largo, apéndice del conectivo de las anteras hasta de $1.5 \mathrm{~mm}$ de largo, ápice agudo a ligeramente mucronulado; gineceo sincárpico, con numerosos carpelos; apéndices carpelares linear-clavados, basalmente purpúreos y con el ápice blanco-amarillento, de 4 a $7 \mathrm{~mm}$ de largo y $0.9 \mathrm{~mm}$ de ancho, ápice redondeado; frutos esféricos, de hasta $3 \mathrm{~cm}$ de diámetro; semillas numerosas, ariladas, elipsoides, de hasta $1 \mathrm{~mm}$ de largo. Foto 3.

Ejemplares de respaldo: Oaxaca: municipio San Mateo del Mar. North side of road to San Mateo del Mar, $0.8 \mathrm{~km}$ east of road to Colonia Cuauhtemoc, approximately $12 \mathrm{~km}$ E of Salina Cruz, 17 September 1993, local name "piquil", A. Novelo \& J. Wiersema 1187 (MEXU); ejemplar de la misma localidad pero sin marcas purpuráceas en el haz y envés de las hojas, A. Novelo \& J. Wiersema 1191 (MEXU); ejemplar joven, sin flores, laguneta west side of small mountain east of road to $\mathrm{Cd}$. Cuauhtemoc, $1.1 \mathrm{~km}$ south from road to San Mateo del Mar, 17 September 1993, local name "baj-rich", A. Novelo \& J. Wiersema 1194 (MEXU); ejemplar mezclado, flores probablemente de $N$. amazonum o de un híbrido entre $N$. amazonum x $N$. novogranatensis, Cd. Cuauhtémoc, laguna Uderrop Piquil, 
Acta Botanica Mexicana (2000), 51: 53-60

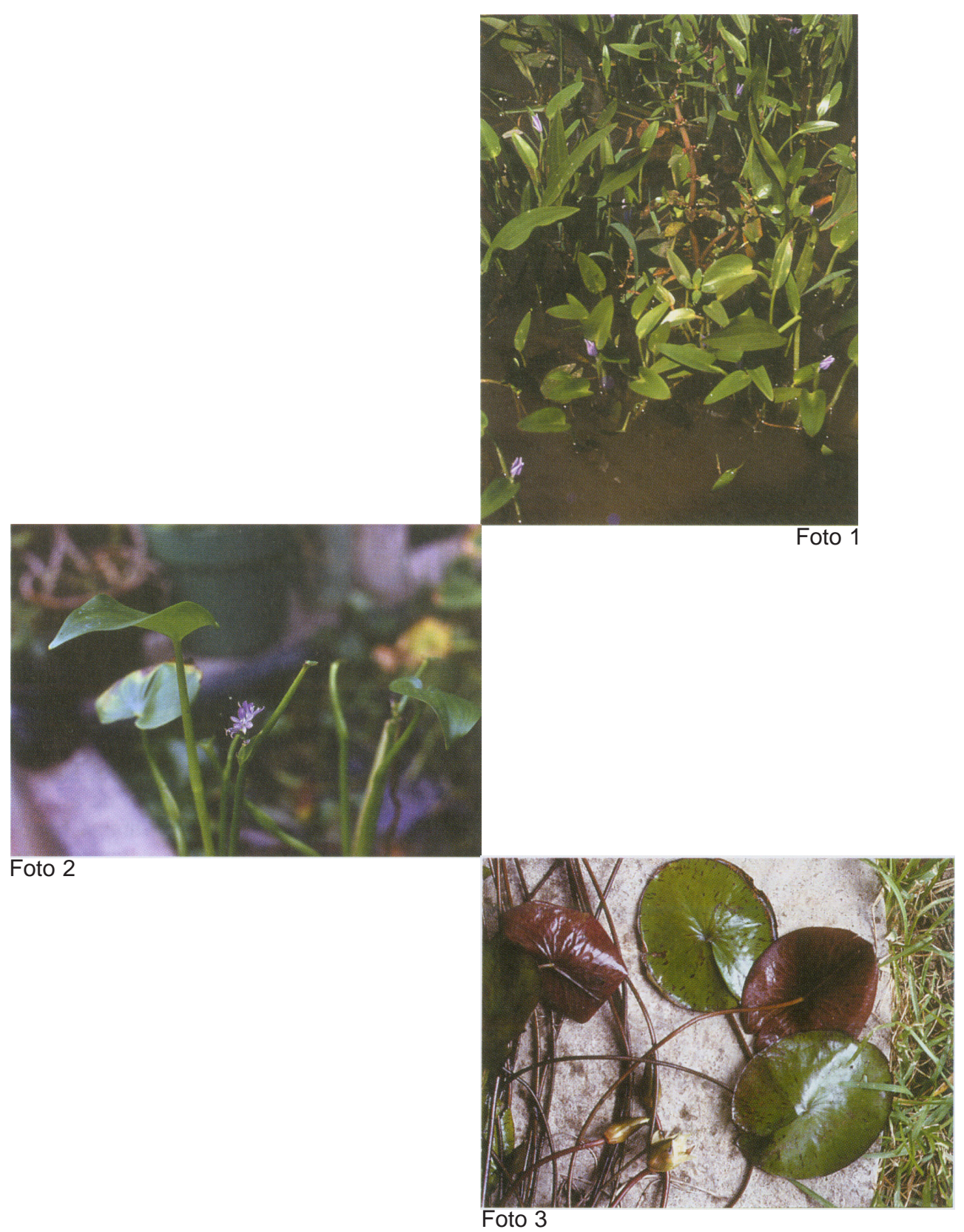

Foto 1. Bergia capensis. Foto 2. Eichhornia paniculata. Foto 3. Nymphaea novogranatensis. 
laguna de agua dulce, 12 septiembre 1978, nombre local "rich piquil", "mjab piquil", $D$. Zizumbo y $P$. Colunga 318 (FCME, MEXU).

Planta propia de charcos efímeros que se forman en las partes bajas y abiertas de los matorrales espinosos dominados por Parkinsonia aculeata. Alt. $5 \mathrm{~m}$. Se le encontró asociada a Nymphaea amazonum, N. jamesoniana y N. pulchella. Especie americana, con anterioridad conocida solamente de Colombia y Venezuela. Elemento escaso y muy localizado en los sitios donde se desarrolla. Se puede considerar como amenazada por el peligro de que su hábitat desaparezca por las actividades humanas.

Varias plantas colectadas en esta zona resultaron ser de características intermedias entre $N$. novogranatensis y $N$. amazonum. El segundo autor cultivó algunas de ellas y las hojas presentaron el típico anillo de pelos alrededor del peciolo en la inserción con el envés de la lámina y los sépalos estaban variegados: dos rasgos distintivos de $N$. amazonum. Sus granos de polen resultaron no ser viables. Por todo lo anteriormente mencionado, esta región Huave resulta ser muy interesante para continuar haciendo estudios sobre hibridación en condiciones naturales en el género Nymphaea.

A continuación se anexa una clave para las especies del subgénero Hydrocallis que se desarrollan en México que puede resultar útil en su identificación.

1 Haz verde, con manchas purpúreas (ocasionalmente sin manchas en $N$. novogranatensis); envés café-rojizo a purpúreo, nervaduras secundarias del envés uniéndose entre sí en forma recta, sin formar una retícula aracnoidea.

2 Flores generalmente imperfectas, tuberíferas

N. prolifera

2 Flores siempre perfectas.

3 La inserción del envés de la hoja con el peciolo presenta un anillo de pelos; ápice de la hoja redondeado

N. amazonum

3 La inserción del envés de la hoja con el peciolo sin un anillo de pelos; ápice de la hoja redondeado a ligeramente mucronulado N. novogranatensis

1 Haz y envés verdes, con o sin pequeñas manchas purpúreas, nervaduras secundarias del envés recurvadas en forma cóncava, formando una retícula aracnoidea.

4 Láminas foliares ovadas a orbiculares, envés generalmente sin manchas purpúreas; apéndices carpelares de más de $7 \mathrm{~mm}$ de longitud N. conardii

4 Láminas foliares elípticas, envés comúnmente con pequeñas manchas purpúreas; apéndices carpelares de menos de $7 \mathrm{~mm}$ de longitud N. jamesoniana

\section{LITERATURA CITADA}

Anónimo. 1970. Carta de climas. Tuxtla Gutiérrez. 15 Q-VII. CETENAL-UNAM. Talleres Gráficos de la Nación. México, D.F.

Cook, C. D. K. 1990. Aquatic plant book. SPB Academic Publishing. The Hague. 229 pp.

García, E. 1964. Modificaciones al sistema de clasificación climática de Koeppen (para adaptarlo a las condiciones de la República Mexicana). Universidad Nacional Autónoma de México. México, D.F. 217 pp. 
Lot, A., A. Novelo y P. Ramírez-García. 1986. Listados florísticos de México: V. Angiospermas acuáticas mexicanas 1. Instituto de Biología, Universidad Nacional Autónoma de México. México, D.F. $60 \mathrm{pp}$.

Lot, A., A. Novelo, M. Olvera y P. Ramírez-García. 1999. Catálogo de angiospermas acuáticas de México. Hidrófitas estrictas emergentes, sumergidas y flotantes. Cuadernos del Instituto de Biología 33. Universidad Nacional Autónoma de México. México, D.F. 161 pp.

Molau, U. 1983. Elatinaceae. In: G. Harling y B. Sparre (eds.). Flora of Ecuador 20: 19-23.

Novelo, A. 1996. Sistemática de la familia Pontederiaceae en México. Tesis de Doctorado. Facultad de Ciencias, Universidad Nacional Autónoma de México. México, D.F. 171 pp.

Novelo, A. y J. Bonilla. 1999. Nymphaeaceae. In: Rzedowski, J. y G. Calderón de Rzedowski (eds.). Flora del Bajío y de Regiones Adyacentes. Fascículo 77. Instituto de Ecología, A.C. Centro Regional del Bajío. Pátzcuaro, Michoacán, México. 13 pp.

Olvera, M. y A. Lot. 1991. Nuevo registro de Nymphaea prolifera (Nymphaeaceae) para México. Bol. Soc. Bot. México 51: 93-94.

Ramírez-García, P. y A. Novelo. 1987. Nymphaea amazonum (Nymphaeaceae) en México; clave de las especies del subgénero Hydrocallis en el país. An. Inst. Biol. Univ. Nac. Autón. Méx. Ser. Bot. 58: 87-91.

Wiersema, J. H. 1987. A monograph of Nymphaea subgenus Hydrocallis (Nymphaeaceae). Syst. Bot. Monogr. 16: 1-112.

Zizumbo, D. y P. Colunga. 1982. Los Huaves, la apropiación de los recursos naturales. Universidad Autónoma Chapingo. Texcoco, Estado de México. 277 pp. 